\title{
Direct observation of a Ga adlayer on a GaN(0001) surface by LEED Patterson inversion
}

\author{
S. H. Xu,${ }^{1}$ Huasheng Wu, ${ }^{1}$ X. Q. Dai, ${ }^{1}$ W. P. Lau, ${ }^{1}$ L. X. Zheng, ${ }^{1}$ M. H. Xie,${ }^{1}$ and S. Y. Tong ${ }^{2}$ \\ ${ }^{1}$ Department of Physics and HKU-CAS Joint Lab on New Materials, The University of Hong Kong, Hong Kong, China \\ ${ }^{2}$ Department of Physics and Materials Science, City University of Hong Kong, Hong Kong, China \\ (Received 18 September 2002; revised manuscript received 27 November 2002; published 17 March 2003)
}

\begin{abstract}
A low-energy electron diffraction (LEED) Patterson function (PF) with multiple incident angles is used to obtain three-dimensional interatomic information of hexagonal $\mathrm{GaN}(0001)$ grown on a $6 H-\mathrm{SiC}(0001)-\sqrt{3}$ $\times \sqrt{3}$ surface. A Ga-Ga atomic pair between the Ga adlayer and the terminating Ga layer is observed in the LEED PF. This provides direct experimental evidence to support the structural model proposed by firstprinciples calculations. The LEED PF also shows that the GaN film has a hexagonal structure and the surface has single-bilayer steps.
\end{abstract}

DOI: 10.1103/PhysRevB.67.125409

PACS number(s): 68.35.Bs, 61.14.Hg, 68.55.Jk

\section{INTRODUCTION}

The symmetry and local atomic structure of a GaN surface grown by molecular-beam epitaxy (MBE) have been extensively studied in recent years. ${ }^{1-7}$ The surfaces of hexagonal GaN films are either Ga terminated (0001) or N terminated $(000 \overline{1})$. From low-energy electron diffraction (LEED), reflection high-energy electron diffraction (RHEED), and scanning tunneling microscopy (STM) studies, it has been shown that both surfaces exhibit a variety of reconstructions, ranging from $(1 \times 1),(2 \times 2)$, and $(5 \times 5)$ to (" $1 \times 1$ "), depending on the Ga concentration on the surface. On the GaN(0001) surface, for example, Smith et al. ${ }^{1-3}$ suggested that two or more additional layers of $\mathrm{Ga}$ are present to explain a pseudo- $1 \times 1$ (or " $1 \times 1$ ") structure. This structure has been further studied by first-principles calculations. ${ }^{6}$ Direct experimental observation of such an additional Ga layer on the surfaces is urgently required to support these models. There have so far been no reports of such experimental observations, to our knowledge.

Recently, a LEED Patterson function (PF) has been used to determine the vector positions of atoms relative to other atoms in the surface region. ${ }^{8-10}$ The method has been extended to include LEED $I$ - $V$ spectra at multiple incident directions and momentum transfers. ${ }^{9,10}$ This technique makes it possible to obtain a three-dimensional (3D) atomic structure from LEED $I-V$ spectra without structural modeling. Because LEED is very sensitive to several surface atomic layers, the PF maps can be used to determine the surface atomic structure of the $\mathrm{GaN}(0001)$ surface.

In this paper, we use the multiple incident angle LEED PF method to determine the structure of the GaN(0001)- $1 \times 1$ surface. The $\mathrm{GaN}(0001)$ film was grown on a $6 H-\operatorname{SiC}(0001)-\sqrt{3} \times \sqrt{3}$ substrate using MBE. The results of the LEED PF maps clearly show a Ga adlayer on top of the last Ga-terminated bilayers. The PF images also show that the epitaxial film has a hexagonal structure and consists of terraces with single bilayer height differences.

\section{EXPERIMENTS}

The experiments were carried out in a multifunctional ultrahigh vacuum (UHV) system that includes chambers for
MBE, STM, LEED, AFM, and x-ray photoemission spectroscopy (XPS). The samples, grown in a MBE chamber, were transferred in situ into LEED and STM chambers for measurements of LEED $I-V$ spectra and observation of the surface morphology, respectively. The $6 H-\mathrm{SiC}(0001)$ substrates (Cree Research, Inc.) were degreased in acetone and alcohol before being transferred into the UHV system. The substrates were heated to $1100^{\circ} \mathrm{C}$ under a flux of Si. This procedure results in a very sharp $(\sqrt{3} \times \sqrt{3}) R 30^{\circ}$ LEED pattern.

On the clean $(\sqrt{3} \times \sqrt{3}) R 30^{\circ} \mathrm{SiC}$ surface, a thick $(\sim 1 \mu \mathrm{m})$ $\mathrm{GaN}$ film was grown at $650{ }^{\circ} \mathrm{C}$ heated by a dc current under a Ga-rich condition. ${ }^{5,7}$ The growth rate is about $0.26 \AA$ s. During the growth, the $\mathrm{Ga}$ and $\mathrm{N}$ source fluxes were supplied, respectively, from a conventional Knudsen cell and from an Oxford plasma unit. The Knudsen cell temperature was $975^{\circ} \mathrm{C}$ while the pressure in the MBE chamber was 5 $\times 10^{-5}$ Torr at a $\mathrm{N}_{2}$ flow rate of 0.13 standard cubic centimeters per minute.

An automated computer program was used to record LEED images at arbitrary incident angles in the energy range (50-402 eV) with a constant energy step of $4 \mathrm{eV}$. In the LEED experiments, a constant suppressor voltage of $4 \mathrm{~V}$ was used for collection of elastically scattered electrons. For the LEED PF inversion, nine incident directions were used. The polar angle $\theta$ was varied from $0^{\circ}$ (normal) to $39^{\circ}$ with a constant step of $13^{\circ}$. The azimuthal angle $\varphi$ was varied from one mirror plane to its nearest neighboring mirror plane (i.e., within one irreducible region). The step of $\varphi$ rotation was varied with the value of $\theta$. Figure 1 presents the schematic geometry of the multiple incident directions $\left(\hat{k}_{i}\right)$. The purpose of this arrangement was to keep an approximate constant solid angle spanned by each direction in one irreducible region. Intensities of the LEED spots $I\left(\hat{k}_{i}, \vec{q}\right)$ were obtained from the LEED images at these incident angles. The momentum transfer is $\vec{q} \equiv \vec{k}_{f}-\vec{k}_{i}$. The LEED PF $P(\vec{R})$ was obtained by a phase sum of the following integral: ${ }^{9}$

$$
P(\vec{R})=\left|\sum_{\hat{k}_{i}} \sum_{\vec{g}_{\|}} \int I\left(\hat{k}_{i}, \vec{g}_{\|}+q_{\perp} \hat{e}_{z}\right) e^{i \vec{q} \cdot \vec{R}} d q_{\perp}\right|^{2},
$$




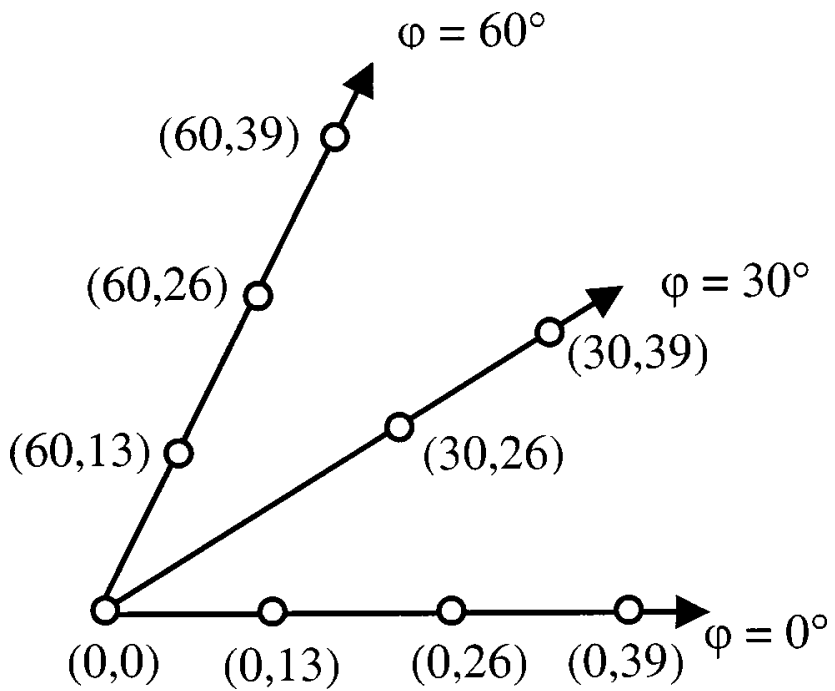

FIG. 1. Two-dimensional schematic map of multiple incident angles arranged from one mirror plane $\left(\varphi=0^{\circ}\right)$ to its nearest neighbor mirror plane $\left(\varphi=60^{\circ}\right)$. The numbers (unit: deg.) inside parentheses are polar angle $\theta$ and azimuthal angle $\varphi$, respectively.

where $\vec{g}_{\|}$is the two-dimensional reciprocal lattice vector and $\vec{q}=\left(\vec{g}_{\|}, q_{\perp} \hat{e}_{z}\right)$. Also, $\hat{e}_{z}$ is a unit vector normal to the surface.

\section{EXPERIMENT RESULTS AND DISCUSSIONS}

The epitaxial film grown by MBE exhibits a $1 \times 1$ structure, as shown in a typical LEED pattern measured at an energy of $70 \mathrm{eV}$ (Fig. 2). Such a $1 \times 1$ structure exists in the whole experimental energy range (50 to $402 \mathrm{eV}$ ) while the background increases with the increase of energy. We do not observe any " $1 \times 1$ " pattern as found by Smith et al. ${ }^{3}$ Figure 3 displays some LEED $I-V$ spectra extracted from LEED images in the normal incident direction. The similarity of beam $(0,1)$ and $(1,0)$ implies that the film has a sixfold rotational symmetry.

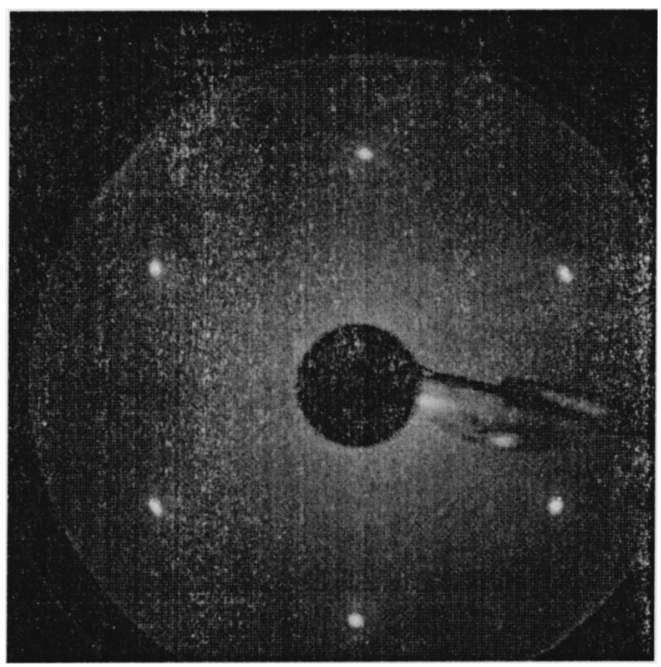

FIG. 2. LEED pattern measured at the energy of $70 \mathrm{eV}$ on a $\mathrm{GaN}(0001)$ surface showing $1 \times 1$ structure.

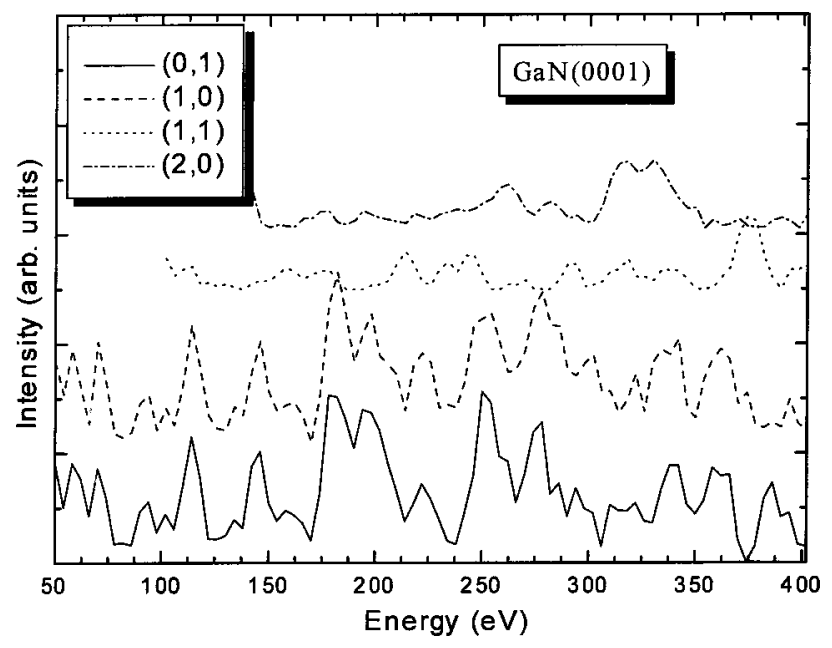

FIG. 3. Selected LEED $I-V$ spectra of a $1 \times 1 \mathrm{GaN}(0001)$ surface at normal incident angle.

The LEED $I$ - $V$ spectra are inverted to obtain the Patterson function $P(\vec{R})$ according to Eq. (1). Figure 4 shows the LEED PF, viewed in the $(11 \overline{2} 0)$ plane, using data from all nine incident angles including about 200 independent beams representing about 9000 independent data points. To suppress spurious noise near the origin, the PF value inside a sphere of radius of $1.5 \AA$ from the origin is set to zero. In this figure, every spot with a vertical distance greater than $1.5 \AA$ in the PF map can be assigned to an atomic pair of the GaN structure. There are, nevertheless, some weak noises in the $\mathrm{PF}$. These noises can possibly be attributed to disorders or defects as a result of the imperfect surface.

Generally speaking, the strongest spots in Fig. 4 come from $\mathrm{Ga}-\mathrm{Ga}$ pairs. The spots from N-Ga or Ga-N pairs are weaker, while spots from N-N pairs are too weak to be observed. ${ }^{11}$ This is because the scattering factor of $\mathrm{N}$ atoms is much smaller than that of $\mathrm{Ga}$ atoms. The strongest spot in Fig. 4 is at $(0,-2.6 \AA)$. This spot comes from the Ga adlayer on top of the last Ga-terminated bilayer. It cannot be assigned to any bulk atomic pair in either hexagonal or cubic $\mathrm{GaN}$. The adlayer is unlikely to be $\mathrm{N}$ because the $\mathrm{N}-\mathrm{Ga}$ nearest neighbor distance is only $1.9 \AA$. This result implies that the surface is Ga polar and that there is one ordered $\mathrm{Ga}$ adlayer on top of the terminating $\mathrm{Ga}$ atoms on $\mathrm{GaN}$ surface. The appearance of the strong spot at $(0,-5.2 \AA)$ clearly demonstrates that the $\mathrm{GaN}$ film is hexagonal, i.e., of the $A B A B \cdots$ stacking sequence. If the film were zinc blende instead, i.e., of the $A B C A B C \cdots$ stacking sequence, there would not be a spot at this position. As a result, the atomic structure of this sample is expected to be that shown in the left half of Fig. 5 as a single-terrace hexagonal structure. The bright spots at depths of $1.9,2.6,3.2$, and $5.2 \AA$ on the $z$ axis obviously correspond to the atomic pairs $7 / 9,2 / 4,4 / 14$, and $4 / 18$ and their equivalent pairs in Fig. 5, respectively. Also the spots at depths of 2.6 and $5.2 \AA$ on the left side of Fig. 4 are due to atomic pairs $4 / 9,2 / 9$, and their equivalent pairs, respectively. 


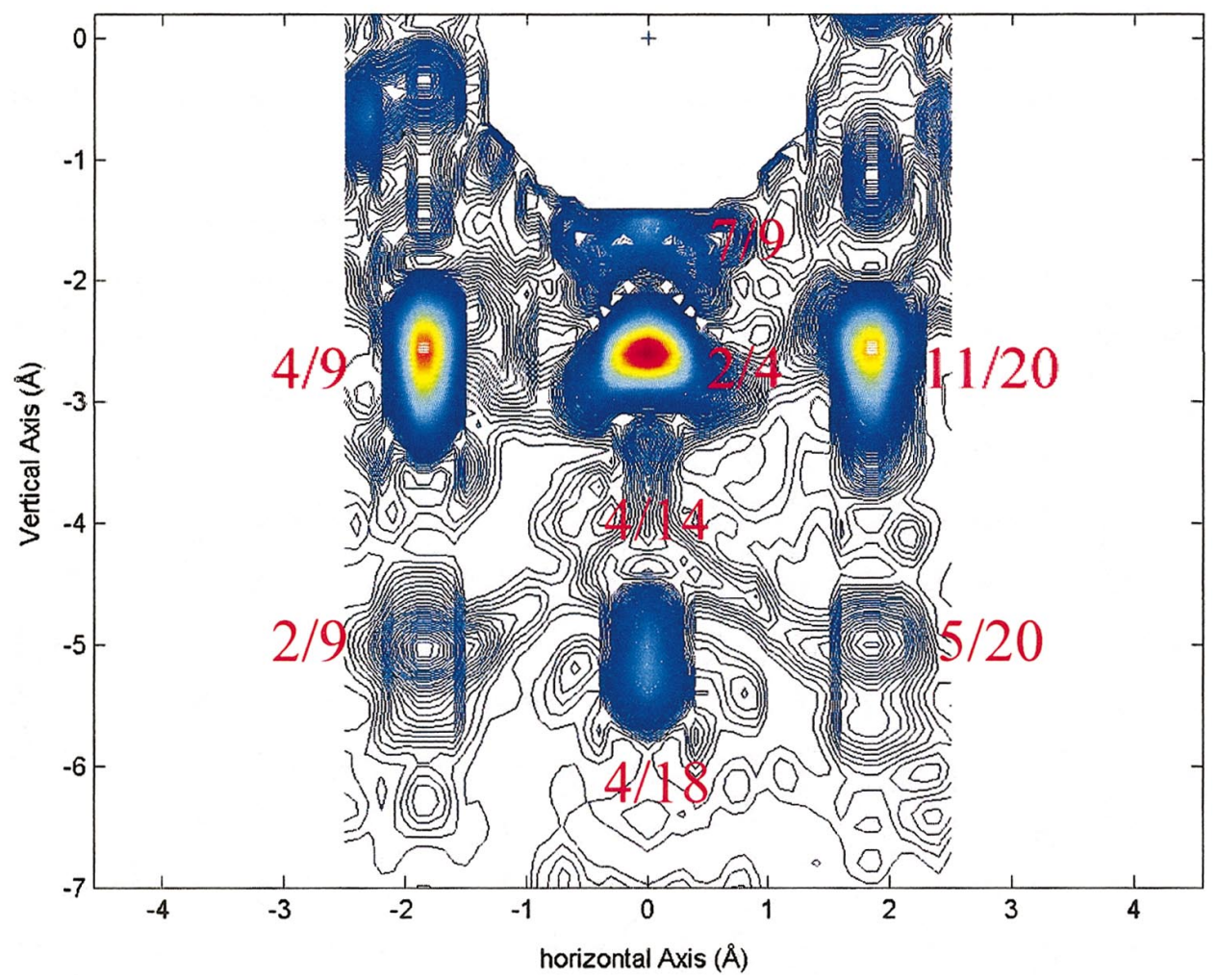

FIG. 4. (Color) LEED PF in the $(11 \overline{2} 0)$ plane from the experimental LEED $I-V$ spectra taken at the nine incident angles shown in Fig. 1. The numbers next to the spots correspond to the atomic pairs shown in Fig. 5.
There are difficulties, unfortunately, in assigning spots on the right side of Fig. 4 with the single-terrace model. In this model, spots at a depth of $2.6 \AA$ would be assigned, in accordance with their positions, to the atomic pair 9/18. This assignment is, however, incorrect given the relevant spot intensities. From Fig. 5, the pair 9/18 is much deeper than the pair 4/9, so the intensity of the former pair should be much weaker than that of the latter pair due to strong electron attenuation. This is not, however, supported by the experiment LEED PF, in which their intensities are almost symmetric. Moreover, the spot at the depth of $5.2 \AA$ on the right side cannot be attributed to any pairs in this model. This difficulty

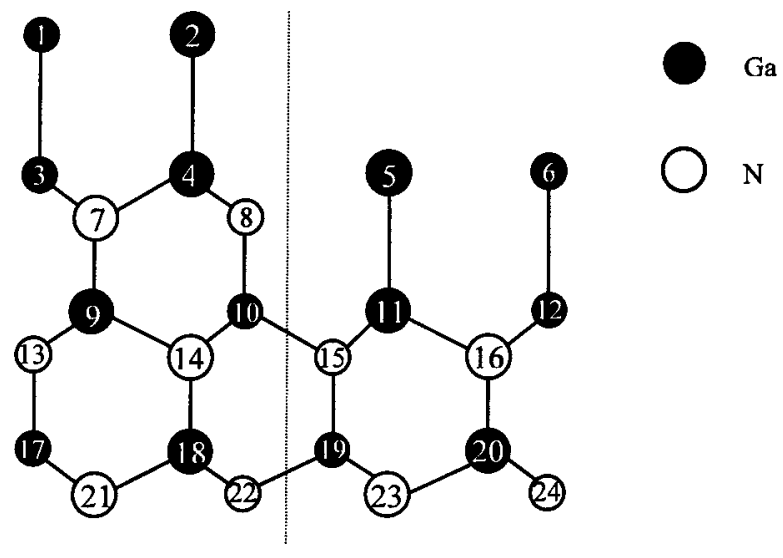

FIG. 5. Schematic diagram of the (one ML) Ga adlayer on a Ga-terminated (0001) surface consisting of two terraces with a bilayer height deference. The filled circles are $\mathrm{Ga}$ atoms, while the

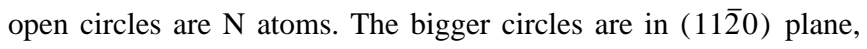
while smaller circles are off this plane. can be overcome by suggesting that there are approximately equal areas of exposed terraces with single bilayer height differences so the neighboring terraces rotate $60^{\circ}$ from each other. This hypothesis agrees with the observation by STM. ${ }^{5,7}$ Under this model, the two spots at depths of 2.6 and $5.2 \AA$ correspond to atomic pairs $11 / 20$ and $5 / 20$, respectively, of the step-down terrace (Fig. 5, right half). Since these two pairs in the second terrace are counterparts of the pairs 4/9 and 2/9 in the first terrace, their intensities are certainly almost symmetric, as shown in the experimental LEED PF.

The appearance of the $2 / 9$ and $5 / 20$ spots provides further evidence that this sample has $\mathrm{Ga}$ termination. Only the Ga-Ga pair can give a strong spot at such a large distance. Finally, the distance of the Ga-Ga pair (i.e., spot 2/4) obtained from the LEED PF map is $2.6 \AA$, which agrees very well with the values of $2.54 \AA$ from the first-principles calculations. ${ }^{6}$ It is noteworthy that the LEED PF shows no evidence of a second $\mathrm{Ga}$ adlayer on the surface, as proposed by the first-principles calculation. ${ }^{6}$ This may be due to the strong disorder in the second Ga adlayer, as suggested by the calculation $^{6}$ and by STM observation. ${ }^{3}$

A final consideration is that such a hexagonal phase always grows on $6 H$-SiC $(0001)-\sqrt{3} \times \sqrt{3}$ substrates even during its early stage deposition or at the interface between the epitaxial film and the substrate. This implies that the $6 H-\operatorname{SiC}(0001)-\sqrt{3} \times \sqrt{3}$ substrate favors the growth of the hexagonal GaN film.

\section{CONCLUSIONS}

Atomic pairs between the Ga adlayer and the terminating Ga-N bilayer have been observed by the experimental LEED 
PF. The PF map provides strong evidence that the film grown on the $6 H-\mathrm{SiC}(0001)-\sqrt{3} \times \sqrt{3}$ surface is Ga-terminated hexagonal GaN. The symmetric intensity in the LEED PF also implies that the surface of the epitaxial film has terraces with single bilayer height differences which is in agreement with the STM observations. ${ }^{5,7}$ No other ordered Ga adatoms were observed on this film.

\section{ACKNOWLEDGMENTS}

The authors would like to thank Ho Win Kin for his most generous help and technical support. Our work was supported in part by grants from Hong Kong's Research Grant Council (HK RGC Grant Nos. HKU7120/00P, HKU7097/ 00P, and HKU7142/99P).
${ }^{1}$ A. R. Smith, R. M. Feenstra, D. W. Greve, J. Neugebauer, and J. E. Northrup, Phys. Rev. Lett. 79, 3934 (1997).

${ }^{2}$ A. R. Smith, R. M. Feenstra, D. W. Greve, M.-S. Shin, M. Skowronski, J. Neugebauer, and J. E. Northrup, Appl. Phys. Lett. 72, 2114 (1998).

${ }^{3}$ A. R. Smith, R. M. Feenstra, D. W. Greve, M.-S. Shin, M. Skowronski, J. Neugebauer, and J. E. Northrup, J. Vac. Sci. Technol. B 16, 2242 (1998).

${ }^{4}$ Qi-Kun Xue, Q. Z. Xue, R. Z. Bakhtizin, Y. Hasegawa, I. S. T. Tsong, and T. Sakurai, Phys. Rev. Lett. 82, 3074 (1999).

${ }^{5}$ M. H. Xie, S. M. Seutter, W. K. Zhu, L. X. Zheng, Huasheng Wu, and S. Y. Tong, Phys. Rev. Lett. 82, 2749 (1999).
${ }^{6}$ J. E. Northrup, J. Neugebauer, R. M. Feenstra, and A. R. Smith, Phys. Rev. B 61, 9932 (2000).

${ }^{7}$ S. M. Seutter, M. H. Xie, W. K. Zhu, L. X. Zheng, Huasheng Wu, and S. Y. Tong, Surf. Sci. 445, L71 (2000).

${ }^{8}$ C. Y. Chang, Z. C. Lin, Y. C. Chou, and C. M. Wei, Phys. Rev. Lett. 83, 2580 (1999)

${ }^{9}$ Huasheng Wu and S. Y. Tong, Phys. Rev. Lett. 87, 036101 (2001).

${ }^{10}$ Huasheng Wu, Shihong Xu, Simon Ma, WP. Lau, MH. Xie, and S. Y. Tong, Phys. Rev. Lett. 89, 216101 (2002).

${ }^{11}$ This statement has been verified by a dynamical LEED $I-V$ calculation. The simulated results show that the intensities of $\mathrm{N}-\mathrm{N}$, $\mathrm{Ga}-\mathrm{N}$, or N-Ga pairs are much weaker than that of $\mathrm{Ga}-\mathrm{Ga}$ pairs. 